\title{
STUDIES OF RIBOSE METABOLISM. III. THE PATHWAY OF RIBOSE CARBON CONVERSION TO GLUCOSE IN MAN ${ }^{1}$
}

\author{
BY HOWARD H. HIATT \\ (From the Departments of Medicine, Harvard Medical School and Beth Israel Hospital, \\ Boston, Mass.)
}

(Submitted for publication November 22, 1957; accepted January 2, 1958)

The conversion of ribose carbon to hexose via the reactions of the pentose phosphate pathway has been observed in studies with rat liver homogenates (1), rat liver slices (2), and in the intact mouse (3). Since most of these reactions are reversible (4), they provide mechanisms not only for ribose conversion to hexose, but for ribose synthesis as well. Indeed, recent work has demonstrated that a human carcinoma cell in tissue culture (5) as well as the intact human subject (6) synthesize ribose via this pathway. This report provides further evidence that in man the reactions of this pathway mediate the interconversion of ribose and glucose. D-Ribose specifically labeled with radioactive carbon was given to a patient with inoperable carcinoma. Isotope distribution in glucose subsequently isolated from this patient's blood is consistent with the conversion of ribose carbon to hexose via the pentose phosphate pathway.

\section{METHODS}

Sixty microcuries of D-ribose-1-C $\mathrm{C}^{14}$ (24 mg.) in 10 $\mathrm{ml}$. of isotonic saline was sterilized by passage through a sintered glass bacteriological filter. This solution was administered intravenously to a 75 year old female three hours after breakfast. The patient was suffering from carcinoma of the head of the pancreas, demonstrated to be inoperable on abdominal laparotomy, but her fasting blood sugar level, renal function, and hepatic parenchymal function (serum thymol turbidity, cephalin flocculation, albumin and globulin, and cholesterol, total and esterified) were normal. Fifteen minutes and two hours following the ribose injection, $50 \mathrm{ml}$. of heparinized blood was removed from an antecubital vein of the opposite arm. The blood was treated with 3 $\mathrm{ml}$. of 70 per cent perchloric acid, and the protein precipitate removed by centrifugation and washed with $10 \mathrm{ml}$. of water. The supernatant solution and wash were brought to $\mathrm{pH} 7.0$ with $5.0 \mathrm{~N} \mathrm{KOH}$, and the insoluble potassium perchlorate removed by centrifugation. The supernatant solution was treated with barium hydroxide and zinc sulfate (7) to remove a small amount of residual

1 This investigation has been aided by grants from the Jane Coffin Childs Memorial Fund for Medical Research and from the National Cancer Institute (C-3151), United States Public Health Service. protein. Following centrifugation the clear supernatant solution was reduced to a volume of approximately $30 \mathrm{ml}$. by lyophilization and then deionized by passage through a mixed bed resin (Amberlite MB-3, Fisher Scientific Co.). Since the effluent contained radioactive material other than glucose, further purification was carried out by phosphorylation of the glucose, adsorption of the sugar phosphate on an ion-exchange resin, washing the phosphate ester free of contaminants, elution of the ester, and, finally, dephosphorylation. One-half of the deionized solution, containing approximately 200 micromoles of glucose, was brought to $\mathrm{pH} 7.6$ with $0.1 \mathrm{~N} \mathrm{KOH}$ and treated with 400 micromoles of adenosine triphosphate (ATP), first brought to $\mathrm{pH} 7.6,100$ micromoles of $\mathrm{MgCl}_{2}$, and $10 \mathrm{mg}$. of crude yeast hexokinase (8). The mixture was stirred magnetically at $25^{\circ} \mathrm{C}$. in a beaker in which external electrodes from a Beckman $\mathrm{pH}$ meter were suspended. The reaction was followed by measuring the quantity of $0.1 \mathrm{~N} \mathrm{NaOH}$ required to maintain the $\mathrm{pH}$, since for every mole of glucose phosphorylated, one mole of acid is produced (8). Following phosphorylation of 82 per cent of the glucose, the mixture was adsorbed on Dowex-1-acetate (the chloride form of the resin is equally satisfactory). The resin was washed with several volumes of water until the effluent was free of radioactivity, and the glucose-6-phosphate was eluted with $0.1 \mathrm{~N} \mathrm{HCl}$. The eluate was neutralized with $1.0 \mathrm{~N}$ $\mathrm{KOH}$, and the volume reduced to $5.0 \mathrm{ml}$. by lyophilization. The $\mathrm{pH}$ was adjusted to 5.0 , and the ester was dephosphorylated by treatment with $10 \mathrm{mg}$. of acid phosphatase at $37^{\circ} \mathrm{C}$. for three hours. The solution was then deproteinized with perchloric acid and deionized as described above. An aliquot of this solution was chromatographed in butanol-acetic acid-water (9), and was found to contain a single radioactive spot on radioautography.

Glucose was degraded by incubation with Leuconostoc mesenteroides (10). The fermentation products were converted to $\mathrm{BaCO}_{3}$ by techniques which have been summarized elsewhere (11), and which permit the isolation of each carbon atom of the glucose molecule free of the others. In order to determine whether added glucose-6phosphate could be degraded by $L$. mesenteroides the sugar phosphate was incubated with a lyophilized preparation of the organism. The lyophilized bacteria fermented glucose actively, but had no discernible effect on the phosphate ester. Thus, the dephosphorylation step was required prior to further degradation of the isolated sugar.

Chemical and enzymatic procedures. Ribose was analyzed by the orcinol procedure (12) and glucose by the method of Nelson (13) and Somogyi (7). Glucose-6- 
phosphate was assayed spectrophotometrically with glucose-6-phosphate dehydrogenase and triphosphopyridine nucleotide (TPN) (14).

Materials. Ribose-1-C $\mathrm{C}^{14}$ was obtained from the $\mathrm{Na}$ tional Bureau of Standards through the courtesy of Dr. H. S. Isbell. Crude yeast hexokinase, glucose-6-phosphate dehydrogenase, TPN, and ATP were purchased from Sigma Chemical Co. Glucose-6-phosphate was purchased from Schwarz Biochemical Co.

Radioactivity determinations were carried out with a "micromil" end-window counter with a counting effciency of approximately 20 per cent. Barium carbonate was counted at "infinite" thickness and all other samples at "infinite" thinness.

\section{RESULTS}

Blood glucose samples, obtained 15 minutes and 2 hours following the injection of ribose- $\mathrm{C}^{14}$, had specific activities of 395 and 133 counts per minute (cpm) per mg., respectively.

The degradation data are presented in Table I. Radioactivity was found predominantly in carbons 1 and 3 of the glucose molecule, with the latter more active than the former. The isotope pattern in the sample isolated at 2 hours was virtually identical with that observed in the 15 minute specimen, and very similar to the distribution of radioactivity in mouse (3) and in rat (15) liver glycogen isolated following ribose-1- $\mathrm{C}^{14}$ administration (Table I).

The unlikely possibility that trace quantities of radioactive ribose remaining in the circulation could have contributed to the isotope pattern can be ruled out as a result of a previously reported investigation (3). In this study a mixture of unlabeled glucose and ribose-1- $\mathrm{C}^{14}$ was degraded by $L$. mesenteroides. No radioactivity was found in carbons 1 or 3 , the sites of major isotope concentration in the blood glucose of the patient given ribose-1- $\mathrm{C}^{14}$.

\section{DISCUSSION}

The fraction of ribose carbon which was converted to blood glucose within the 15 minutes following ribose administration may be calculated from the product of the specific activity of the blood glucose [395 cpm per mg.], the blood glucose concentration [90 $\mathrm{mg}$. per $100 \mathrm{ml}$.], and the glucose space [24 per cent (16) of the patient's body weight, 50 kilograms]. This figure, $4.80 \times$ $10^{8} \mathrm{cpm}$, which represents 16 per cent of the administered radioactive carbon, is a minimal one, for it does not include the glucose catabolized during the 15 minutes following ribose administration. Six to fifteen per cent of the administered radioactivity was found in liver glycogen of mice given ribose-1-C ${ }^{14}(3)$.

Studies with plants, bacteria and animals suggest a uniformity in the pathway of conversion of ribose to hexose (4). The pentose must first be phosphorylated, and a specific kinase for this reaction has been isolated from mammalian liver (17).

1) Ribose + ATP $\rightarrow$ ribose-5-phosphate + ADP (adenosine diphosphate)

This pentose phosphate may then be converted to hexose phosphate via the nonoxidative series of reactions of the pentose phosphate pathway:

2)
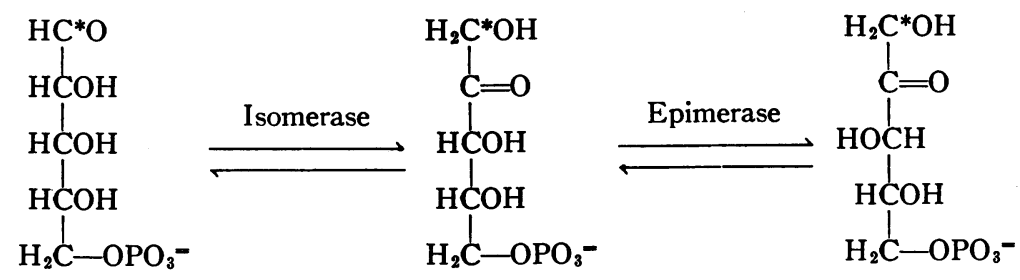

Ribose-5-P

Ribulose-5-P

Xylulose-5-P
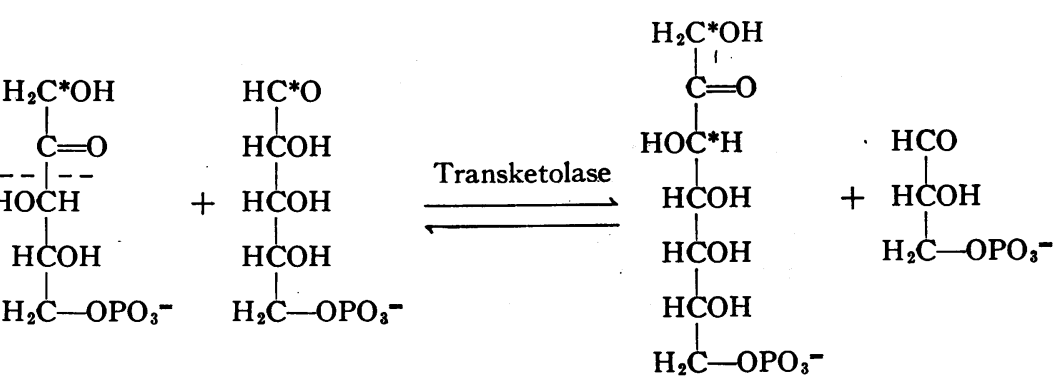

Xylulose $\mathrm{P}$

Ribose P

Sedoheptulose $\mathrm{P}$

Triose $\mathrm{P}$ 
4)
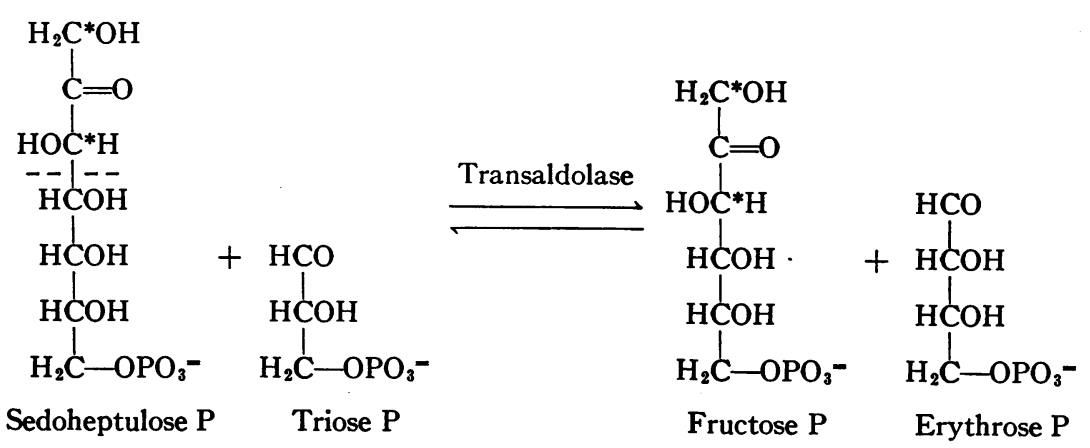

5)

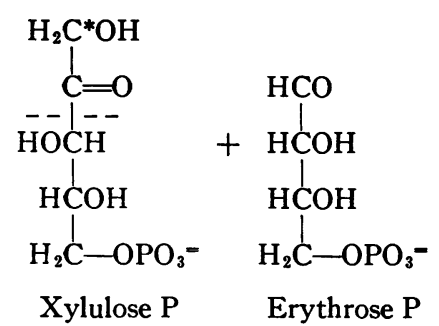

To summarize these reactions, three molecules of ribose phosphate would give rise to two molecules of fructose phosphate and one of triose phosphate. Radioactivity, originally introduced in the first carbon atom of pentose, is denoted by an asterisk in reactions 2 through 5 . In reaction 3 the first two carbons of xylulose phosphate are transferred to ribose phosphate. Thus, carbon 1 (C-1) of the product, sedoheptulose phosphate, is derived from the first carbon of one molecule of pentose and carbon 3 from $\mathrm{C}-1$ of the other. Since in reaction 4 , the first three carbons of fructose-6-phosphate are derived from the first three carbons of sedoheptulose, then carbons 1 and 3 of hexose originate from carbon 1 of ribose. Hence, if hexose is synthesized from ribose via these reactions, radioactivity introduced in position 1 of ribose should appear in carbons 1 and 3 of hexose. Such was indeed found to be the case in the blood glucose isolated from the patient in- jected with ribose-1-C $\mathrm{C}^{\mathbf{1 4}}$ (Table I); this glucose was labeled almost exclusively in carbons 1 and 3 . The striking similarity of isotope distribution in this glucose with mouse and rat hexose synthesized from ribose carbon is consistent with ribose to hexose conversion via the same pathway in all three species.

The mechanism which accounts for the predominance of isotope in carbon 3 as compared to carbon 1 has been discussed in detail elsewhere (3). To summarize briefly, it has been shown that fructose-6-phosphate as well as xylulose phosphate may act as the donor of "active glycolaldehyde," the term applied to the two-carbon fragment transferred in the transketolase reaction (reaction 3) (18). If ribose-1- $\mathrm{C}^{14}$-phosphate were the acceptor in this reaction and unlabeled fructose phosphate were the donor, sedoheptulose-3-C $\mathrm{C}^{14}$-phosphate would be the product:
6)

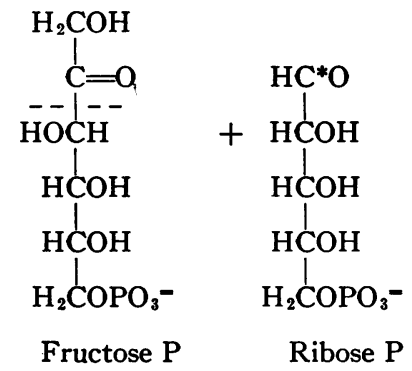

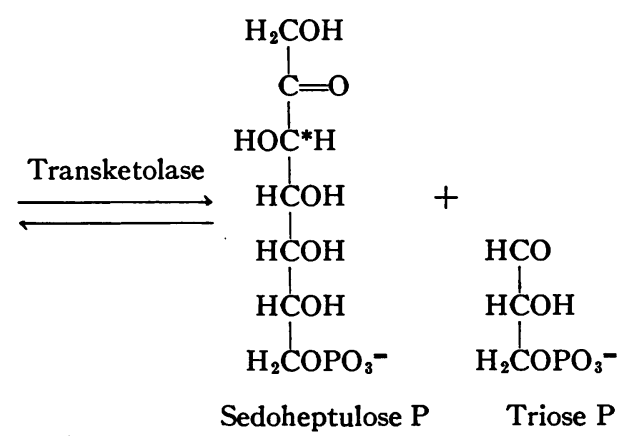


TABLE I

Distribution of radioactivity in human blood glucose and in animal liver glycogen after administration of ribose-1-C $C^{14}$

\begin{tabular}{|c|c|c|c|c|c|c|}
\hline \multirow[b]{2}{*}{ Sample } & \multicolumn{6}{|c|}{$\begin{array}{l}\text { Per cent total activity in glucose } \\
\text { carbon atoms }\end{array}$} \\
\hline & C-1 & C-2 & C-3 & C-4 & C-5 & C-6 \\
\hline $\begin{array}{l}15 \text { minute glucose } \\
2 \text { hour glucose } \\
\text { Mouse glycogen } \\
\text { Rat glycogen } \\
\text { 15 }\end{array}$ & $\begin{array}{l}30.1 \\
31.4 \\
34.0 \\
32.7\end{array}$ & $\begin{array}{l}0.9 \\
1.0 \\
3.5 \\
2.7\end{array}$ & $\begin{array}{l}66.7 \\
65.4 \\
52.0 \\
58.2\end{array}$ & $\begin{array}{l}1.5 \\
1.4 \\
4.5 \\
2.5\end{array}$ & $\begin{array}{l}0.4 \\
0.4 \\
3.0 \\
1.7\end{array}$ & $\begin{array}{l}0.4 \\
0.4 \\
3.0 \\
2.2\end{array}$ \\
\hline
\end{tabular}

The transfer of the first three carbons of sedoheptulose-3- $\mathrm{C}^{14}$ to triose phosphate in the transaldolase reaction (reaction 4 ) would result in the production of hexose-3-C ${ }^{14}$-phosphate. Since in our study the ribose was administered in tracer quantity, nonradioactive fructose phosphate, a normal intracellular component, was undoubtedly present in large quantity relative to that xylulose-1- $\mathrm{C}^{\mathbf{1 4}}$ phosphate derived from ribose-1- $\mathrm{C}^{\mathbf{1 4}}$ in reaction 2. Hence, the amount of available unlabeled "active glycolaldehyde" (derived from fructose phosphate) was considerably greater than the amount of radioactive "active glycolaldehyde" (derived from xylulose-1-C ${ }^{14}$-phosphate). In the transketolase reaction the acceptor molecule was ribose$1-\mathrm{C}^{14}$, which transferred its radioactivity to position 3 of the product (reactions 3,4 , and 6 ). The isotope concentration in carbon 1 of the hexose product, however, was diluted to an extent dependent on the proportion of nonradioactive to radioactive "active glycolaldehyde" participating in the reaction. Evidence supporting this hypothesis has been presented in the previously cited report (3).

\section{SUMMARY}

Ribose specifically labeled with radioactive carbon was administered to an elderly female with advanced malignant disease. A method is described for the isolation of blood glucose. The isotope pattern in blood glucose isolated following the administration of ribose- $1-\mathrm{C}^{14}$ is consistent with the transformation of ribose carbon to glucose via the reactions of the pentose phosphate pathway.

\section{ACKNOWLEDGMENT}

The valuable technical assistance of Miss Jacqueline Lareau is gratefully acknowledged.

\section{REFERENCES}

1. Horecker, B. L., Gibbs, M., Klenow, H., and Smyrniotis, P. Z. The mechanism of pentose phosphate conversion to hexose monophosphate. I. With a liver enzyme preparation. J. biol. Chem. 1954, 207, 393.

2. Katz, J., Abraham, S., Hill, R., and Chaikoff, I. L. The occurrence and mechanism of the hexose monophosphate shunt in rat liver slices. J. biol. Chem. 19.55, 214, 853.

3. Hiatt, H. H. Glycogen formation via the pentose phosphate pathway in mice in vivo. J. biol. Chem. 1957, 224, 851.

4. Horecker, B. L., and Mehler, A. H. Carbohydrate metabolism. Ann. Rev. Biochem. 1954, 24, 207.

5. Hiatt, H. H. Studies of ribose metabolism. I. The pathway of nucleic acid ribose synthesis in a human carcinoma cell in tissue culture. $\mathrm{J}$. clin. Invest. 1957, 36, 1408.

6. Hiatt, H. H. Pathways of ribose synthesis in normal and pentosuric human subjects (abstract). J. clin. Invest. In press.

7. Somogyi, M. Determination of blood sugar. J. biol. Chem. 1955, 160, 69.

8. Colowick, S. P., and Kalckar, H. M. An activator of the hexokinase system. J. biol. Chem. 1941, 137, 789.

9. Partridge, S. M. Filter-paper partition chromatography of sugars. Biochem. J. 1948, 42, 238.

10. Gunsalus, I. C., and Gibbs, M. The heterolactic fermentation. II. Position of $\mathrm{C}^{\mathbf{1 4}}$ in the products of glucose dissimilation by Leuconostoc mesenteroides. J. biol. Chem. 1952, 194, 871.

11. Hiatt, H. H., Goldstein, M., Lareau, J., and Horecker, B. L. The pathway of pyruvate conversion to hexose in muscle. J. biol. Chem. 1958, 231, 303.

12. Albaum, H. G., and Umbreit, W. W. Differentiation between ribose-3-phosphate and ribose-5-phosphate by means of the orcinol-pentose reaction. J. biol. Chem. 1947, 167, 369.

13. Nelson, N. A photometric adaptation of the Somogyi method for the determination of glucose. J. biol. Chem. 1944, 153, 375.

14. Kornberg, A., and Horecker, B. L. Glucose 6-phosphate dehydrogenase in Methods of Enzymology, S. P. Colowick and N. O. Kaplan, Eds. New York, Academic Press Inc., 1955, vol. 1, p. 323.

15. Hiatt, $H . H$. Unpublished observation.

16. Shreeve, W. W., Baker, N., Miller, M., Shipley, R. A., Incefy, G. E., and Craig, J. W. $\mathrm{C}^{14}$ studies in carbohydrate metabolism. II. The oxidation of glucose in diabetic human subjects. Metabolism 1956, 5, 22.

17. Agranoff, B. W., and Brady, R. O. Purification and properties of calf liver ribokinase. J. biol. Chem. 1956, 219, 221.

18. Racker, E., de la Haba, G., and Leder, I. G. Transketolase-catalyzed utilization of fructose 6-phosphate and its significance in a glucose 6-phosphate oxidation cycle. Arch. Biochem. 1954, 48, 238. 